\title{
Semanta - Semantic Email in Action
}

\author{
Simon Scerri, Ioana Giurgiu, Brian Davis, and Siegfried Handschuh
}

Digitial Enterprise Research Institute, National University of Ireland Galway, IDA Business Park, Galway, Ireland \{simon.scerri, ioana.giurgiu, brian.davis, siegfried.handschuh\}@deri.org

\begin{abstract}
Semanta is a system supporting Semantic Email, implemented as an add-in to two popular Mail User Agents, using existing email transport technology and integrated with the Social Semantic Desktop. It enables machines to support email users with correctly interpreting, handling and keeping track of action items within email messages, visualizing email workflows, and extracting tasks and appointments from email messages.
\end{abstract}

Keywords: Semantic Email, Email Workflows, Speech Act Theory.

\section{Introduction}

Recent surveys have shown that about $80 \%$ of knowledge workers prefer emails as a business communication tool ${ }^{1}$. However email has been known to present knowledge management problems for its users, who become overwhelmed with the tasks they need to perform and keep track of, eventually resulting in their failure to keep up with the ongoing email workflows whilst gaining a loss in productivity. We do not restrict email use, but support its management by providing structure and semantics to email's characteristic ad-hoc workflows. In supporting these workflows, we support the users. In our approach, the content of an email message can be summarized into a number of Action Items (e.g. Meeting Request, Task Assignment, File Request etc.). Once exchanged, every single action item can be seen as the start, or continuation of a separate workflow. We have presented the theoretical ideas behind the sMail (Semantic Email) Framework in detail in previous work [1], and demonstrated [2] how Semantic Email can perform an important role in the social fabric of the Social Semantic Desktop [3]. We will now demonstrate the usefulness of its implementation - Semanta, via a common email exchange scenario between two knowledge workers, after an insight into related work and the system's architecture.

\section{Related Work}

The notion of Semantic Email was originally introduced by Dowell et. al. [4] to refer to an email message consisting of a structured query coupled with corresponding

${ }^{1}$ http://www.highbeam.com/doc/1G1-100628406.html 
explanatory text, based on a number of Semantic Email Processes that represent commonly occurring workflows within email. The system, successfully implemented within Mangrove [5], provided templates which exposed structured knowledge about these scenarios to both humans and machines. Semanta is more flexible, since although we do provide templates, our approach is more oriented towards ad-hoc email processes, i.e. we support email workflows as they evolve, without restricting the user's actions whatsoever. We also apply Speech Act Theory [6] to provide more adequate representation of email action items. Although the same theory was applied to email beforehand - particularly to address the problems of email task management [7][8] and email classification [9][10][11]; it was never combined with semantic technologies. We represent action items by speech act instances from within the sMail Ontology ${ }^{2}$. Furthermore, by grounding Semanta within the Nepomuk ${ }^{3}$ Social Semantic Desktop, semantic knowledge obtained from email is integrated with that on the semantic desktop so that other applications can re-use it.

\section{Semanta}

The following is a very brief overview of Semanta's architecture, which consists of four main components:-

- Text Analytics Service - provides semi-automatic annotation of email content by employing Ontology-Based Information techniques via the use of the GATE Framework [12] and the Jape Patterns Engine [13]

- Social Semantic Desktop - provides an RDF store that Semanta can read and write to, and the conceptual modelling required for Semantic Email data to be integrated in the Semantic Desktop knowledge base.

- Simplistic Graphical User Interface - abstracts theoretical models and semantics from the user beneath add-ins to popular Mail User Agents (Outlook, Thunderbird).

- Semantic Email Service - Exposes knowledge in the sMail Framework to Semanta and serves as the link between the GUI and the other components

\section{Scenario}

We highlight the support for ad-hoc email workflows provided by Semanta via a typical email exchange scenario ${ }^{4}$ between two (more participants are supported) hypothetical colleagues - Claudia and Dirk. Claudia proceeds to write an email via Microsoft Outlook, her preferred email client. When she is done, Semanta attempts automatic annotation of action items in the content, and finds two action items:

1. "How is the review report going?" - an Information Request addressed to Dirk

2. "Can we discuss the review tomorrow afternoon?" - a Meeting Request between Claudia and Dirk addressed to Dirk

\footnotetext{
${ }^{2}$ http://ontologies.smile.deri.ie/smail\#

${ }^{3}$ http://www.nepomuk.semanticdesktop.org/

${ }^{4}$ More demonstrations available at http://smile.deri.ie/projects/semanta/\#Demonstrations
} 
Although Claudia is happy with these annotations, she can change or create annotations via the Semanta Annotation Wizard. The latter assists the user with the intuitive, step-by-step construction or modification of semantic annotations representing action items (Fig. 1). When Claudia sends the email, metadata is invisib ly transported alongside the email content in the email headers. When Dirk receives the email, Semanta detects action items within and flags it appropriately in the inbox. After opening it, Dirk can react to the action items via a right mouse click. Depending on its type, the user is given a number of appropriate options. In Fig. 2, Dirk has reacted to the Information Request by providing the required information ("So far, so good"). As regards the Meeting Request apart from approving, ignoring, declining or amending it, Dirk can react in any other way via the 'Other..' option. In fact Dirk wants to question the purpose of the meeting. The annotation wizard is shown right away so that Dirk can choose an action (e.g. Request Information) and provide text for this action ("What is there to be discussed?"). This constitutes an additional action item in this ad-hoc email workflow. When Dirk closes the incoming email, its semantic representation on his desktop is updated, and a reply email is automatically sent back to Claudia.

Claudia replies to Dirk's question and although he acknowledges her reply, he forgets to reconsider her meeting request. This is a frequent email problem hindering collaborative work, but it can be addressed by Semanta's Action Item Tracker. Dirk uses the tracker to view all action items he received but still needs to process, via the 'Pending Incoming Item' view. One of these items represents Claudia's Meeting Request (Fig. 3). Action items are shown in the context of the workflow that they belong to. In the 'Context' pane on the right, Dirk can see that he reacted to this request by asking for more information regarding the event ("What is there to be discussed?"). He also sees that although Claudia replied to the latter request ("The way forward!"), he forgot to reconsider the meeting proposal. After making up his mind, he opens the containing email directly from the tracker and accepts the Meeting Request.

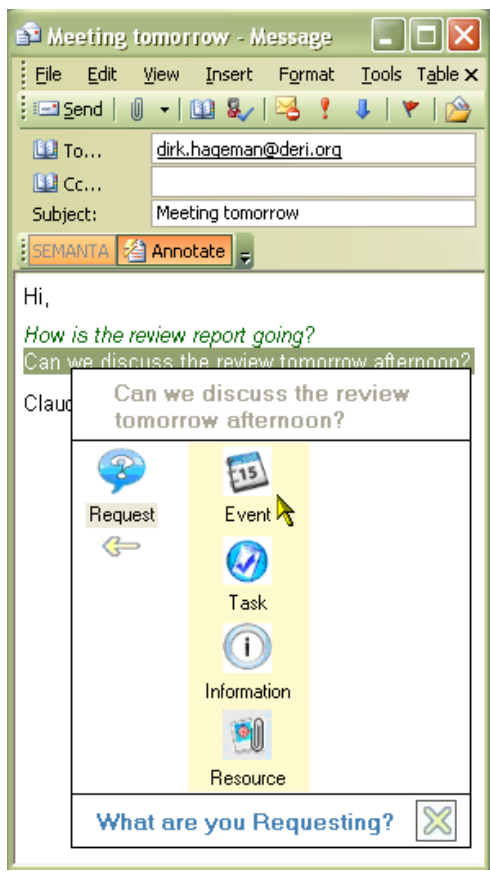

Fig. 1. Annotation Wizard

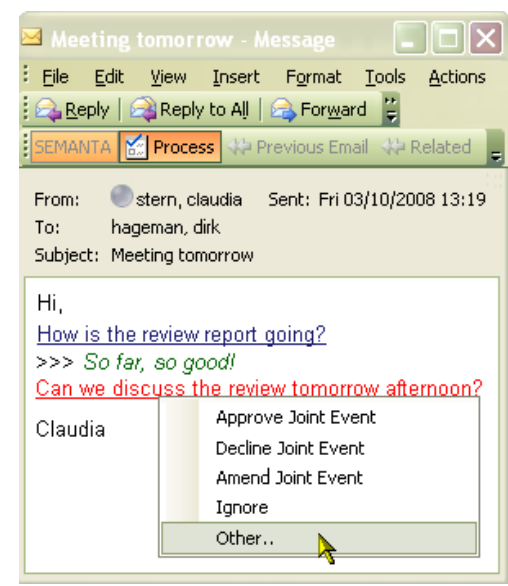

Fig. 2. Processing Incoming Email 


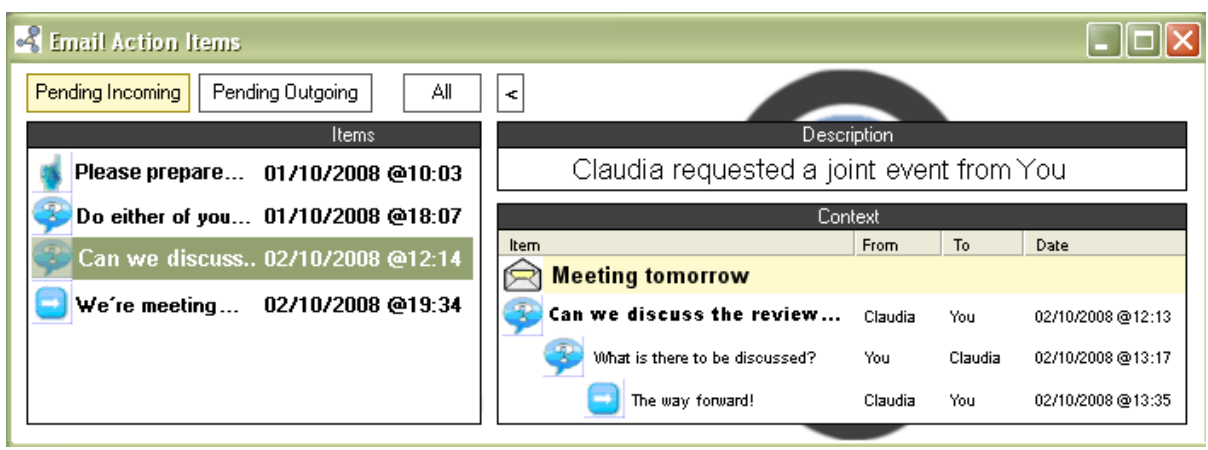

Fig. 3. Semanta's Action Item Tracker, visualizing email workflows

When Dirk accepts the meeting, Semanta detects that an event involving Dirk has been generated (Fig. 4). Dirk can either dismiss detected events/tasks, or add them to his Outlook Calendar/Tasklist. Dirk chooses to add the event to his calendar, whereby the semi-populated, native Outlook Appointment Item creation window is brought up. The subject of this appointment item is obtained from the text of the original Meeting Request followed by the text of Dirk's Meeting Approval. The meeting attendees for this appointment are known given the semantic annotation for the Meeting Request as shown in annotation 2 at the start of our scenario. Dirk's meeting approval also triggers an automatic email reply notifying Claudia that her meeting proposal has been approved. When Claudia reads this email, she is also assisted with the addition of this event to her calendar.

The objects involved in this email exchange and their relationships are represented semantically using the Social Semantic Desktop ontologies ${ }^{5}$, e.g. the emails (nmo:Email), the action items (smail:SpeechAct), the appointment item (ncal:Event), Claudia/Dirk (nco:Contact), etc. In Fig. 3 we saw how semantic relationships between action items in email workflows and their relationship to the messages used to carry out these workflows can be utilised for the user's benefit. Semanta provides three other useful func-

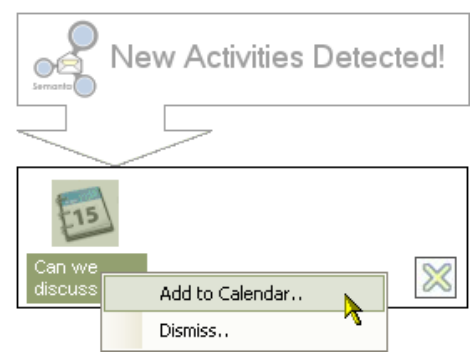

Fig. 4. Event Detection tions - the 'Related Email' button directly loads the email that generated the appointment from within the appointment item window; the 'Related Activity' button points back to the generated appointment from within the email; and the 'Previous Email' button enables Dirk to traverse up the email thread.

\section{Conclusion}

We have demonstrated how Semanta - a fully-implemented system supporting Semantic Email, can assist the user with the execution of previously implicit ad-hoc

\footnotetext{
${ }^{5}$ http://www.semanticdesktop.org/ontologies/
} 
email workflows. By exposing knowledge about email messages, contacts involved, action items within and the workflows they generate, resulting events and tasks, as well as their relationships to machines, the user can be assisted with better managing their email use. Furthermore, the integration of Semanta within the Social Semantic Desktop means that the aggregated semantic knowledge is immediately exposed to other desktop applications. The results of an initial evaluation [14] show that its support for semantic email processes is applicable to everyday email usage, and that it does not constrain the conventional ways in which people tend to use email.

Acknowledgments. The work presented in this paper was supported (in part) by the Lion project supported by Science Foundation Ireland under Grant No. SFI/02/CE1/I131 and (in part) by the European project NEPOMUK No FP6-027705.

\section{References}

1. Scerri, S., Mencke, M., Davis, B., Handschuh, S.: uating the Ontology underlying sMail the Conceptual Framework for Semantic Email Communication. In: Proc. Language Resources and Evaluation 2008, Marrakech, Morocco (2008)

2. Scerri, S., Handschuh, S., Decker, S.: Semantic Email as a Communication Medium for the Social Semantic Desktop. In: Bechhofer, S., Hauswirth, M., Hoffmann, J., Koubarakis, M. (eds.) ESWC 2008. LNCS, vol. 5021, pp. 124-138. Springer, Heidelberg (2008)

3. Decker, S., Martin, F.: The Social Semantic Desktop. Technical Report (2004)

4. McDowell, L., Etzioni, O., Halevey, A., Levy, H.: Semantic Email. In: Proc. World Wide Web (2004)

5. McDowell, L., Etzioni, O., Gribble, S., Halevey, A., Levy, H., Pentney, W., Verma, D., Vlasseva, S.: Evolving the Semantic Web with Mangrove. UW Tech Report (2003)

6. Searle, J.: Speech Acts. Cambridge University Press, Cambridge (1969)

7. Khoussainov, R., Kushmerick, N.: Email task management: An iterative relational learning approach. In: Conference on Email and Anti-Spam, CEAS 2005 (2005)

8. Corston-Oliver, S., Ringger, E., Gamon, M., Campbell, R.: Task-focused summarization of email. In: Text Summarization Branches Out workshop, ACL 2004 (2004)

9. Khosravi, H., Wilks, Y.: Routing email automatically by purpose not topic. Natural Language Engineering 5, 237-250 (1999)

10. Goldstein, J., Sabin, R.E.: Using Speech Acts to Categorize Email and Identify Email Genres. In: System Sciences, HICSS 2006 (2006)

11. Carvalho, V., Cohen, W.: On the collective classification of email speech acts. In: SIGIR 2005, pp. 345-352 (2005)

12. Cunningham, H., Maynard, D., Bontcheva, K., Tablan, V.: GATE: A Framework and Graphical Development Environment for Robust NLP Tools and Applications. In: Proc. 40th Anniversary Meeting of the Association for Computational Linguistics (ACL 2002), Philadelphia (2002)

13. Cunningham, H., Maynard, D., Tablan, K.: JAPE: a Java Annotation Patterns Engine, 2nd edn. Technical report CS-00-10, University of Sheffield, Department of Computer Science (2000)

14. Scerri, S., Davis, B., Handschuh, S., Hauswirth, M.: Semanta - Semantic Email Technology for the Masses. In: Proc. ESWC 2009, Crete, Greece (2009) 